\title{
The exploding-reflector concept for ground-penetrating-radar modeling
}

\author{
José M. Carcione $\left({ }^{1}\right)$, Laura Piñero Feliciangeli $\left({ }^{2}\right)$ and Michela Zamparo $\left({ }^{1}\right)$ \\ ${ }^{(1)}$ Istituto Nazionale di Oceanografia e di Geofisica Sperimentale (OGS), Sgonico, Trieste, Italy \\ ${ }^{2}$ ) Instituto de Ciencias de la Tierra, Departamento de Geofísica, Universidad Central de Venezuela, \\ Caracas, Venezuela
}

\begin{abstract}
The simulation of a stacked radargram requires the calculation of a set of common-source experiments and application of the standard processing sequence. To reduce computing time, a zero-offset stacked section can be obtained with a single simulation, by using the exploding-reflector concept and the so-called non-reflecting wave equation. This non-physical modification of the wave equation implies a constant impedance model to avoid multiple reflections, which are, in principle, absent from stacked sections and constitute unwanted artifacts in migration processes. Magnetic permeability is used as a free parameter to obtain a constant impedance model and avoid multiple reflections. The reflection strength is then implicit in the source strength. Moreover, the method generates normal-incidence reflections, i.e. those having identical downgoing and upgoing wave paths. Exploding reflector experiments provide correct travel times of diffraction and reflection events, in contrast to the plane-wave method.
\end{abstract}

Key words exploding reflector - zero-offset section GPR modeling

\section{Introduction}

The computation of synthetic seismograms for simulating zero-offset (stacked) seismic sections, and the reverse time migration of these sections, requires the use of the explodingreflector concept (Loewenthal et al., 1976; Carcione et al., 1994) and the so-called nonreflecting wave equation (Baysal et al., 1984). This non-physical modification of the wave equation implies a constant impedance model to avoid multiple reflections, which are, in principle, absent from stacked sections and

Mailing address: Dr. José M. Carcione, Istituto Nazionale di Oceanografia e di Geofisica Sperimentale (OGS), Borgo Grotta Gigante 42c, 34010 Sgonico, Trieste, Italy; e-mail: jcarcione@ogs.trieste.it constitute unwanted artifacts in migration processes (Baysal et al., 1983).

The increasing use of Ground-Penetrating Radar (GPR) for solving a wide range of engineering and environmental problems has been facilitated by the application of standard seismic techniques, such as multi-fold coverage and processing (Fisher et al., 1992; Pipan et al., 1996). Modeling, as an interpretation tool, and migration methods are two of the most important processing techniques. In particular, it is necessary to migrate events generated by nearsurface dipping reflectors and buried elements, such as boulders and localized inhomogeneities. In many cases, the radargrams can also be contaminated by surface events generated by trees and metallic objects. Therefore, it is important to develop the concepts of exploding reflector and non-reflecting wave equations for GPR applications.

There are two efficient ways for simulating a zero-offset synthetic survey avoiding calculation of common-shot records: the plane- 
wave method and the exploding reflector method. Both approaches involve a single calculation with a time-domain modeling algorithm. The first consists in sending a horizontal localized plane-wave front down from the surface and recording the response of the subsurface model back at the surface. The travel times obtained with this method are not those obtained with the standard processing sequence. A simple example is given by a single diffraction point at $(0, z)$. If $v$ is the phase velocity of the medium, the travel time at offset $(x, 0)$ is

$$
t=z / v+\sqrt{x^{2}+z^{2}} / v
$$

while a source and a receiver at $(x, 0)$ imply a travel time

$$
t=2 \sqrt{x^{2}+z^{2}} / v
$$

The method is also approximate for dipping layers.

In the exploding-reflector method (Baysal et al., 1984; Carcione et al., 1994), each reflection point in the subsurface explodes at $t=0$ with a magnitude proportional to the normalincidence reflection coefficient. The equation is a modification of the wave equation, where the impedance is constant over the whole model space. In this way, non-physical multiple reflections are avoided and the recorded events are primary reflections. Moreover, the method generates normal-incidence reflections, i.e. those having identical downgoing and upgoing wave paths. In order to obtain the two-way travel time, the phase velocities are halved. Due to sampling constraints, halving the velocities implies doubling the number of grid points. Therefore, the method is less efficient than the plane-wave approach.

In the seismic case, the density is used as a free parameter to obtain a constant impedance model and avoid multiple reflections. From the analogy between acoustic and electromagnetic waves (Carcione and Cavallini, 1995), the density is mathematically analogous to the magnetic permeability. We scale this property by a factor depending on the permittivity, to obtain a model where all the media have the same electromagnetic impedance. In addition, the condition that the phase velocity remains unchanged also requires the scaling of the permittivity and the conductivity. In an example, we consider the Transverse-Magnetic (TM) wave equations and compare synthetic radargrams computed with both the plane-wave and the exploding-reflector methods. The numerical solver used here consists of the pseudospectral Fourier method for computing the spatial derivatives, and a Runge-Kutta method for time integration (Carcione, 1996). However, any other full-wave equations method, finitedifferences, pseudospectral, or finite-elements, can be used.

\section{Maxwell's equations}

Maxwell's equations for isotropic media and time harmonic fields with time dependence $\exp (i \omega t)$, where $\omega$ is the angular frequency, read

$$
\nabla \times \boldsymbol{E}=-i \omega \mu_{0} \boldsymbol{H}+\boldsymbol{M}
$$

and

$$
\nabla \times \boldsymbol{H}=i \omega \varepsilon \boldsymbol{E}+\sigma \boldsymbol{E}+\boldsymbol{J}_{s}=i \omega \varepsilon^{*} \boldsymbol{E}+\boldsymbol{J}_{s}
$$

where the symbol $\times$ denotes the vector product, $\boldsymbol{E}$ is the electric field, $\boldsymbol{H}$ is the magnetic field, $\boldsymbol{J}_{s}$ is the electric source, $\boldsymbol{M}$ is the magnetic source, $\mu_{0}$ is the magnetic permeability of vacuum (appropriate for GPR applications), $\varepsilon$ is the dielectric permittivity, $\sigma$ is the con-ductivity, and

$$
\varepsilon^{*}(\omega)=\varepsilon-\frac{i}{\omega} \sigma
$$

is the complex permittivity (Chew, 1990).

\section{The exploding reflector}

Implementation of the exploding reflector method in GPR modeling implies the following:

1) A source is placed at every point on the subsurface interface of interest.

2) The phase velocity of each medium is halved, to obtain the correct two-way travel time 
and amplitude decay for every diffraction and reflection event.

3) The source strength is proportional to the normal-incidence reflection coefficient at each point on the interface (a zero-offset raypath is normal to the reflecting interface).

4) We require that the electromagnetic impedance be the same for all media. Because the algorithm generates non-physical events (the downgoing waves) and approximates a zero-offset stacked section, we must avoid multiple reflections.

We propose the following approximations to meet requirements (2.1) to (3.1):

1) The algorithm used here solves the electromagnetic equations in the time domain, and it is based on a grid method for computing the spatial derivatives. This implies a discretization of the model space. Then, at every grid point of each interface a source with the same time history is initiated at time $t=0$.

2) The phase velocity is given by

$$
V_{p}=\left[\mathfrak{R}\left(\frac{1}{V}\right)\right]^{-1}
$$

where $\mathfrak{R}$ is the real-part operator, and

$$
V=\frac{1}{\sqrt{\mu_{0} \varepsilon^{*}}}
$$

is the complex velocity (Carcione, 1996). Halving the phase velocity can be achieved by the following substitution:

$$
\mu_{0} \rightarrow 4 \mu_{0}
$$

Since the attenuation factor is

$$
\alpha=-\omega \Im\left(\frac{1}{V}\right)
$$

(Carcione, 1996), where $\mathfrak{I}$ is the imaginary-part operator, eq. (3.3) ensures that the amplitude decay corresponds to that of the two-way travel path.

3) The normal-incidence reflection coefficient between medium 1 and medium 2 is given by

$$
R=\frac{I_{2}-I_{1}}{I_{2}+I_{1}}
$$

where

$$
I=\mu V
$$

is the electromagnetic impedance (Chew, 1990, p. 48), with $\mu=\mu_{0}$ here. Equation (3.5) is the reflection coefficient for the electric field of the TE or TM waves. The corresponding normalincidence reflection coefficient for the magnetic field of the TE or TM waves has the opposite sign. The source strength should be proportional to $|R|$. Since $R$ depends on the frequency $\omega$, this requirement cannot be satisfied for all frequencies when using a time-domain solver. In this case, we consider the source strength to be proportional to $\left|R\left(\omega_{d}\right)\right|$, where $\omega_{d}$ is the dominant frequency of the source.

4) We can avoid normal-incidence multiple reflections if all the media have the same electromagnetic impedance. This is evident from eq. (3.5). In order to do this we must scale the material properties accordingly, that is, substitute $\mu_{0}, \varepsilon$ and $\sigma$ with new properties $\widetilde{\mu}, \widetilde{\varepsilon}$ and $\widetilde{\sigma}$. The complex impedance can be made constant by using the Perfectly Matched Layer (PML) method introduced by Berenguer (1994); i.e. using a complex magnetic permeability. However, this approach gives a different complex velocity compared to eq. (3.2). We may impose that the «instantaneous» impedance is the same for all the media. The instantaneous impedance is defined as

$$
I^{\infty}=I(\omega=\infty)
$$

which corresponds to the unrelaxed response of the medium $(t=0)$. In this limit $\sigma / \omega \rightarrow 0$, in general. Then, we assume

$$
I^{\infty}=\sqrt{\frac{\tilde{\mu}}{\tilde{\varepsilon}}}=k
$$

with $k$ a real constant. Moreover, we must impose that the complex velocity (3.2) remains unchanged after introduction of the scaled pro- 
perties, i.e.

The choice

$$
\mu_{0} \varepsilon^{*}=\tilde{\mu}\left(\tilde{\varepsilon}-\frac{i}{\omega} \tilde{\sigma}\right) .
$$

$$
k=I_{0}=\sqrt{\frac{\mu_{0}}{\varepsilon_{0}}}
$$

(the impedance of vacuum) implies

$$
\tilde{\mu}=\frac{\mu_{0}}{a}, \quad \tilde{\varepsilon}=a \varepsilon \quad \text { and } \quad \tilde{\sigma}=a \sigma
$$

where

$$
a=\sqrt{\frac{\varepsilon_{0}}{\varepsilon}} .
$$

Then, $I^{\infty}=I_{0}$ and eq. (3.9) is satisfied.

\section{Example}

We assume an isotropic medium, propagation in the $(x, z)$-plane, and that the material properties and source characteristics are constant with respect to the $y$-coordinate. The radargrams are computed in the space-time domain. Let us denote by $\mathcal{E}$ and $\mathcal{H}$ and $\mathcal{J}$ and $\mathcal{M}$ the corresponding time-domain electric and magnetic fields and sources, and for convenience, the medium properties are indicated by the same symbols, in both, the time and the frequency domains. Under these conditions, $\mathcal{E}_{x}, \mathcal{E}_{z}$ and $\mathcal{H}_{y}$ are decoupled from $\mathcal{E}_{y}, \mathcal{H}_{x}$ and $\mathcal{H}_{z}$, and the first three fields obey the TM wave differential equations

$$
\begin{gathered}
\frac{\partial \varepsilon_{z}}{\partial x}-\frac{\partial \varepsilon_{x}}{\partial z}=\mu \frac{\partial \mathcal{H}_{y}}{\partial t}+\mathcal{M}_{y} \\
-\frac{\partial \mathcal{H}_{y}}{\partial z}=\sigma \varepsilon_{x}+\varepsilon \frac{\partial \varepsilon_{x}}{\partial t}+\mathcal{J}_{s x} \\
\frac{\partial \mathcal{H}_{y}}{\partial x}=\sigma \varepsilon_{z}+\varepsilon \frac{\partial \varepsilon_{z}}{\partial t}+\mathcal{J}_{s z} .
\end{gathered}
$$

The set of properties $(\mu, \varepsilon, \sigma)$ is equal to $\left(\mu_{0}, \varepsilon\right.$, $\sigma)$ for the plane-wave method, and to $\left(\widetilde{\mu}_{0}, \widetilde{\varepsilon}, \widetilde{\sigma}\right)$ for the exploding-reflector method.

Let us consider the model shown in fig. 1. The ray-paths represented with solid lines are

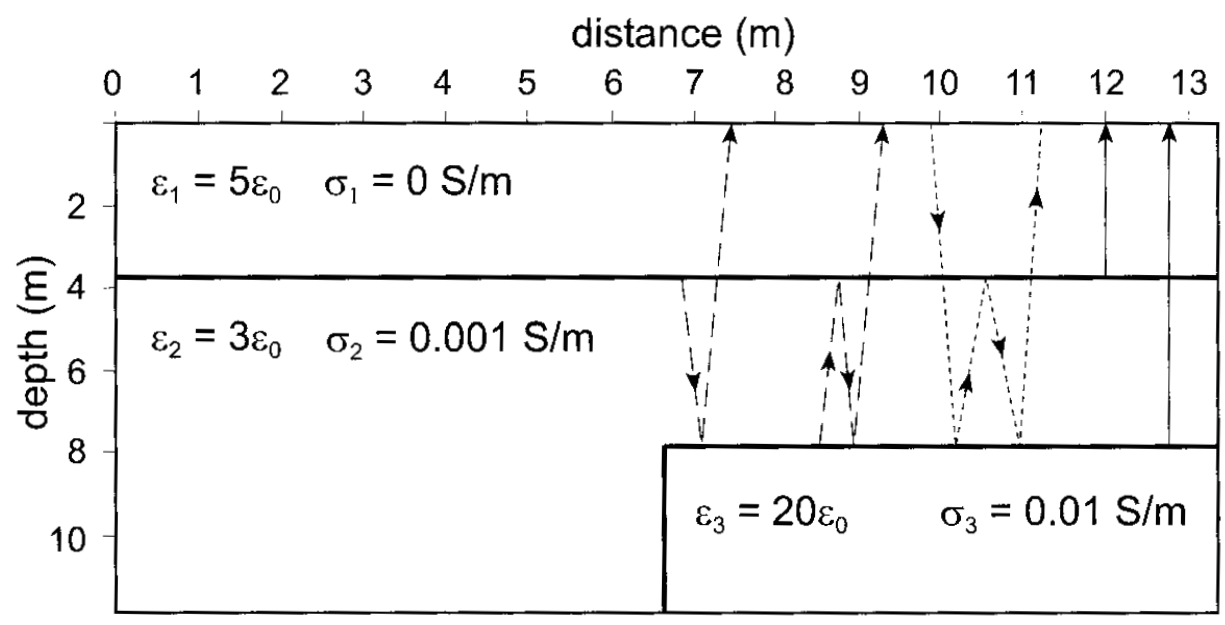

Fig. 1. Model and electromagnetic properties. The ray-paths represented with solid lines are the direct arrivals. The dotted line is a physical multiple. The dashed lines represent non-physical multiple events due to the presence of down-going waves in the exploding-reflector experiment (Carcione et al., 1994). 

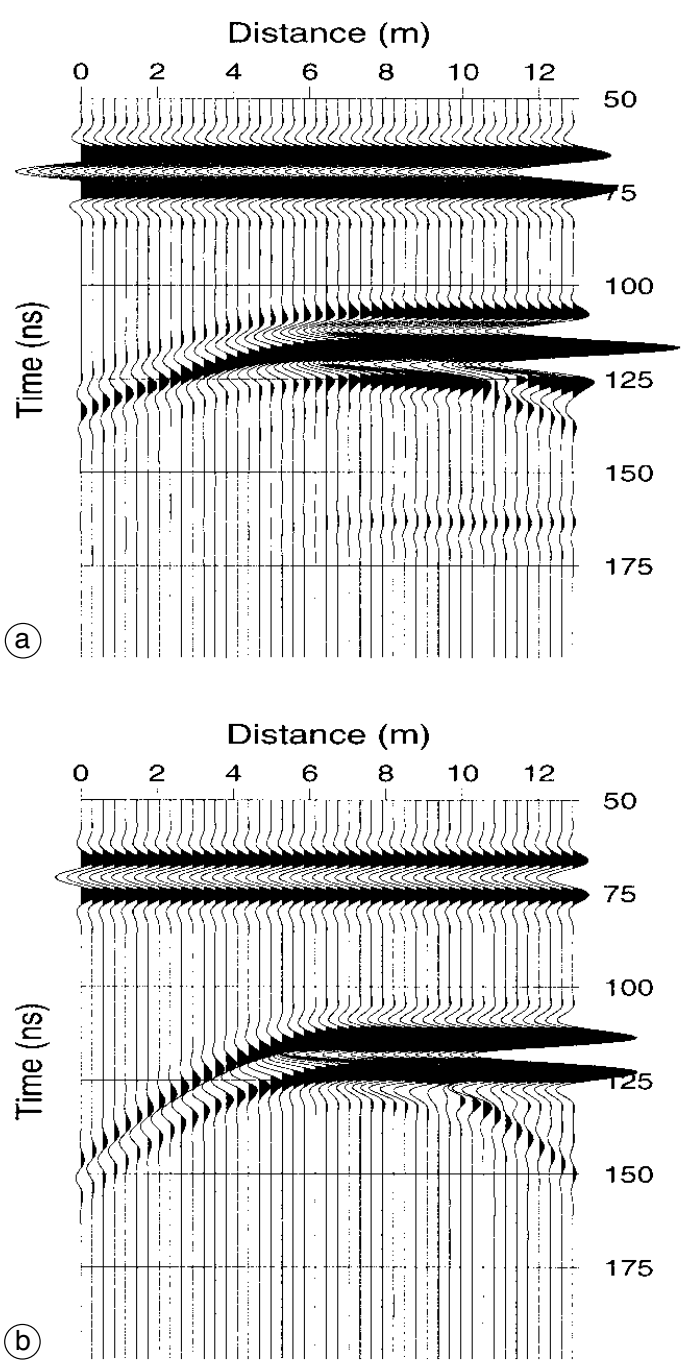

Fig. 2a,b. Synthetic radargrams of magnetic field component, computed with the plane-wave method (a) and the exploding-reflector method (b) (Carcione et al., 1994).

the direct arrivals. The dotted line is a physical multiple, present in the plane-wave response (at $150 \mathrm{~ns}$ ). The dashed lines represent non-physical multiple events due to the presence of downgoing waves in the exploding-reflector experiment (with arrival times of 148 and 194 ns, respectively). The latter events do not appear in the synthetic radargrams because the medium is electromagnetically «transparent» due to the requirement (3.1). The quality factor is given by

$$
Q=\frac{\operatorname{Re}\left(V^{2}\right)}{\operatorname{Im}\left(V^{2}\right)}=\frac{\omega \varepsilon}{\sigma}
$$

(Carcione and Cavallini, 1995). Using $\varepsilon_{0}=8.85$ $10^{-12} \mathrm{~F} / \mathrm{m}$, the quality factors at $100 \mathrm{MHz}$ of the media in fig. 1 are $Q_{1}=\infty, Q_{2}=28$ and $Q_{3}=11$. The numerical mesh for the plane-wave experiment has $N_{X}=N_{Z}=125$ grid points, with a grid spacing $D_{X}=D_{z}=15 \mathrm{~cm}$. The source is a horizontal electric current $\left(\mathcal{J}_{s x}\right)$, whose time history is a Ricker-type wavelet with a central frequency $f_{c}=100 \mathrm{MHz}$ and a cut-off frequency of $200 \mathrm{MHz}$. The Nyquist criterion implies

$$
D_{\max }=\frac{V_{p \min }}{4 f_{c}}
$$

where $D_{\max }$ is the maximum allowed grid size, and $V_{p \min }$ is the minimum phase velocity. The minimum phase velocity is that of medium 3 (see fig. 1), with an unrelaxed (optical) value of $6.7 \mathrm{~cm} / \mathrm{ns}$. Note that a maximum spacing of $16.77 \mathrm{~cm}$ is allowed. Because the velocities are halved in the exploding-reflector method, we use $D_{X}=D_{z}=7.5 \mathrm{~cm}$, and $N_{X}=N_{z}=243$. The algorithm, similar to that developed by Carcione (1996), is based on the Fourier method for computing the spatial derivatives and a RungeKutta algorithm for the time evolution of the wave field. We use a time step of 0.05 ns. Figure $2 a, b$ shows the synthetic radargrams of the magnetic-field component, obtained with the plane-wave method and the exploding-reflector method, respectively (the pictures have been scaled to obtain comparable amplitudes for the step-interface response). The differences in travel times of the diffraction hyperbolae are evident. The exploding-reflector travel times are greater than the plane-wave travel times, as indicated in the Section 1. Moreover, there is a change of polarity in the plane-wave response of the step. The weak event that peaks at $162 \mathrm{~ns}$ in fig. 2a is the multiple indicated by a dotted line in fig. 1. Note that the non-physical multiples (dashed lines in fig. 1) are absent in fig. $2 b$. 


\section{Conclusions}

We have developed the exploding-reflector method for GPR applications. It provides the correct travel times of diffraction and reflection events, in contrast to the plane-wave method. Moreover, unlike one-way equations, the method can simulate ray-paths which turn around via refraction in the presence of large velocity gradients. The use of a nearly constant impedance condition avoids the non-physical multiple events caused by the presence of down-going waves. The method can be used to approximate stacked radargrams with a single calculation, and in migration algorithms, where it is necessary to avoid interlayer reverberations.

\section{Acknowledgements}

L. Piñero Feliciangeli thanks the «Consejo de Desarrollo Científico y Humanístico» of $\mathrm{UCV}$ for partially funding the research.

\section{REFERENCES}

BAYSAL, E., D.D. KosLOFF and J.W.C. SHERWOOD (1983): Reverse time migration, Geophysics, 48, 1514-1524.

BAYSAL, E., D.D. KOSLOFF and J.W.C. SHERWOOD (1984): A two-way nonreflecting wave equation, Geophysics, 49, 132-141.

BERENGUER, J.P. (1994): A perfectly matched layer for the absorption of electromagnetic waves, J. Comput. Phys., 114, 185-200.

CARCIONE, J.M. (1996): Ground radar simulation for archaeological applications, Geophys. Prospect., 44, 871-888.

CARCIONE, J.M. and F. CAVALLINI (1995): On the acousticelectromagnetic analogy, Wave Motion, 21, 149-162.

Carcione, J.M., G. BöHM and A. Marchetti (1994): Simulation of a CMP seismic section, J. Seism. Explor, 3, 381-396.

CHEW, W.C. (1990): Waves and fields in inhomogeneous media (Van Nostrand Reinhold, New York), pp. 637.

FisHER, E., G.A. MCMECHAN and P. ANNAN (1992): Acquisition and processing of wide-aperture ground-penetrating radar data, Geophysics, 57, 495-504

LOEWENTHAL, D., L. LU, R. ROBERSON and J.W.C. SHERWOOD (1976): The wave equation applied to migration, Geophys. Prospect., 24, 380-399.

PIPAN, M., I. FINETTI and F. FERIGO (1996): Multi-fold GPR techniques with applications to high-resolution studies: two case histories, J. Environ. Eng. Geophys., 1, 83-103.

(received January 10, 2002;

accepted March 13, 2002) 